\title{
Emulating Human Leg Impairments and Disabilities on Humanoid Robots Walking
}

\author{
Sébastien Lengagne, Abderrahmane Kheddar, Sébastien Druon and Eiichi Yoshida
}

\begin{abstract}
In this paper, we present a method for emulating human walking motions with leg impairments or disabilities using humanoid robots. Our optimal dynamic multi-contact motion software generates the emulated motions. We take into account the full-body dynamic model of the robot and consider possible leg impairments as additional physical constraints in the optimization problem. The proposed approach is verified using HRP-2 humanoid robot. Simulations and experiments revealed extra energy consumption when constraints translating impairments are added, which is assessed using an infrared camera. Our research is the first step toward potential usage of humanoid robots in human-centric studies, diagnosis of human disabled motions, as well as an active mannequin for welfare instrument evaluation.
\end{abstract}

\section{INTRODUCTION}

In this paper, we use our on-going work on optimal dynamic multi-contact motions generation [1], [2] to investigate whether adding simple constraints that translate -to some extent- a given human leg impairment, results in emulating a similar walking behavior by a humanoid robot, namely the HRP-2.

There are many efficient on-line methods to generate walking motions for humanoid robots, e.g. [3], [4], [5]. These methods compute the walking motion in two separate stages: the first one consists in generating the trajectory for the Center Of Mass (COM) from a Zero Moment Point (ZMP) trajectory representing the walking motion based on a simplified inverted pendulum model; the second stage deals with the whole body motion generation that tracks at best the COM generated at the first stage while compensating the difference between the simple model and the multi-body humanoid structure.

This type of (over-)simplified method cannot be used when we desire to study motions resulting from such constraints as leg impairments or disabilities because: (i) some hypotheses that are made on the COM, namely the height, may not be known (ii) we may need to apply constraints on walking a priori and not a posteriori, and (iii) following the reason (ii), we may consider a full-body model in order to take into account all the physical limits of the robot, the desired contact stances and possibly additional constraints to reproduce leg disabilities (Fig. 1).

In our work, we plan the motion using a whole body model to generate three walking motions. The first one reproduces a normal walk and is to serve as a comparative reference.

All the authors are with the CNRS-AIST Joint Robotics Laboratory (JRL), UMI3218/CRT, Tsukuba, Japan. A. Kheddar and S. Druon are also with the Interactive Digital Human group of the LIRMM, UMR 5506 Université Montpellier II - CNRS, Montpellier, France.
The second motion emulates the walking of someone who sprained its left knee and wear a splint that prevents the knee joint from moving. As the third motion, we consider that the force applied during the walking on a given leg should not excess a given threshold, which may be the case of a broken or painful foot. To study the impact of those impairments on the walking motion we use infrared recording of the robot in order to localize any extra-energy consumption, and compare the contact forces and knee joint torques obtained from experiments with the HRP-2 robot.

This work is motivated by the application of humanoid robots to human study and diagnosis. If we are able to parameterize the disabled motions, we can reproduce different impairments to understand and diagnose their consequences. Another application we have in mind is the evaluation of human-centric welfare instruments. The proposed method allows the designers of those instruments to evaluate them by simulating various disabled human motions by using the humanoid as an active mannequin.

Section II presents our walking motion generation algorithm that is based on optimization techniques. We then present the additional constraints used to reproduce leg impairments and/or disabilities and the results from the new optimization formulation in Section III. The experimental results with the HRP-2 robot are presented in Section IV.

\section{MOTION GENERATION}

In this Section, we briefly recall the method presented in [6] to generate full-body optimal dynamics multi-contact motion for humanoid robots and virtual avatars and intro-

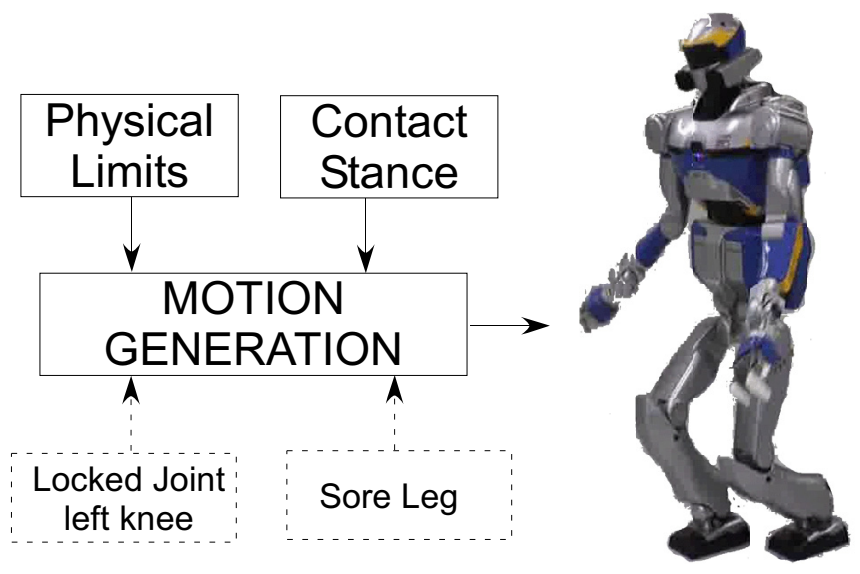

Fig. 1. Motion generation software takes into account the physical limits, the desired contact stances. It is enhanced with additional constraints so as to emulate, on the HRP-2 robot, actual human impaired leg motions. 
duce the basics in terms of terminology, notations, and the general formulation of the motion generation problem as an optimization problem.

\section{A. Optimization problem}

The motion generation problem can be tackled as an optimal control problem [7], [8] that is simply equivalent to the optimization problem that consists in finding the best set of joint trajectories $q(t)$ that solves:

$$
\begin{array}{cc}
\underset{q(t)}{\operatorname{argmin}} & C(q(t)) \\
\forall i, \forall t \in\left[\Delta_{i}\right] & g_{i}(q(t)) \leq 0 \\
\forall j, \forall t \in\left[\Delta_{j}\right] & h_{j}(q(t))=0 \\
\forall t_{k} \in\left\{t_{1}, t_{2}, \ldots\right\} & z_{k}\left(q\left(t_{k}\right)\right) \leq 0
\end{array}
$$

where:

- $C$ is the cost function, which accounts for quantitative or/and qualitative achievements. Well-known costs functions usually used in robotics are: motion duration [9], minimum torque [10], global energy consumption [11], jerk for smooth motion [12], torque change [13], or any weighted combination of the above and more.

- $g$ is the set of continuous inequality constraints, which usually translates the physical limits of the system (joint position, velocity, torques, balance, etc.) that must be satisfied over the whole motion duration, decomposed into several time-intervals $\Delta_{i}$,

- $h$ is the set of continuous equality constraints, e.g. those which define the position of a link in contact with the environment over a time-interval $\Delta_{j}$,

- $z$ is a set of discrete inequality constraints, e.g. those that can be used to specify the position of one part of the robot at a given time,

\section{B. Resolution}

The problem of Eq. (1) is also called an Infinite Programming problem (IP), which can be turned into Semi-Infinite Programming (SIP) using parameterization of the joint trajectories. Inspired from prior work in robotics, e.g. [14], we made the choice to shape the joint trajectories with clamped uniform cubic B-Spline curves:

$$
q_{i}(t)=\sum_{j=1}^{m} b_{j}^{3}(t) p_{i, j}
$$

In order to deal with the time continuous inequality and equality constraints $(g, h)$, which include generally the dynamic, kinematic and geometric models in function of the robot's state variables, we use a time interval decomposition based on Taylor expansions, which result in polynomial approximations of all constraints and the cost function. The Taylor expansions also apply to the states variables of the robots (joint and Cartesian position, velocity and acceleration, contact forces and joint torques) in various algebraic operations [1], and we use additional coefficients in order to compute the contact forces [2].

Note that the coefficients of the time-polynomials (approximating the constraints and the cost function) are function of the optimization variables (the control points of the BSpline). The motion is decomposed into several contact phases and the full-duration motion is generated by finding the best parameter set $\mathbf{P} \in \mathbb{R}^{N}$ such that:

$$
\begin{array}{cl}
\underset{\mathbf{P}}{\operatorname{argmin}} & C(\mathbf{P}) \\
\forall i, \forall t \in\left[\Delta_{i}\right] & g_{i}(\mathbf{P}, t) \leq 0 \\
\forall j, \forall t \in\left[\Delta_{j}\right] & h_{j}(\mathbf{P}, t)=0 \\
\forall t_{k} \in\left\{t_{1}, t_{2}, \cdots, t_{n}\right\} & z_{k}\left(\mathbf{P}, t_{k}\right) \leq 0
\end{array}
$$

with:

$$
\mathbf{P}=\left[p_{1,1}, p_{1,2}, \ldots, p_{N_{d o f}, N_{p}}, \beta_{1,1}, \ldots, \beta_{N_{p}, N_{c}}, T_{1} \ldots, T_{N_{p}}\right]
$$

here, $p_{i, j}$ is the $j^{t h}$ control point of joint $i, \beta_{i, j}$ is the force coefficient of the contact $i$ of phase $j, T_{i}$ is the time duration of phase $i, N_{d o f}$ the number of Degrees Of Freedom (DOF), $N_{p}$ the number of phases and $N_{c}$ the number of contacts for one phase. Using this formulation of the problem, we use the optimization solver IPOPT [15] to get the optimal motion parameters $\mathbf{P}$.

\section{Modeling}

We model humanoid robots as an articulated chain of rigid-bodies and consider the following dynamics equation:

$$
\left[\begin{array}{l}
\Gamma \\
0
\end{array}\right]=\left[\begin{array}{c}
M_{1}(q) \\
M_{2}(q)
\end{array}\right] \ddot{q}+\left[\begin{array}{c}
H_{1}(q, \dot{q}) \\
H_{2}(q, \dot{q})
\end{array}\right]+\left[\begin{array}{c}
J_{1}^{T}(q) \\
J_{2}^{T}(q)
\end{array}\right] \mathbf{F}_{\mathbf{c}}
$$

where $q$ is a vector containing the $n$ joint position $\left(q_{i}\right)$ plus the position and orientation of the reference body, $\Gamma$ is the $n$ vector of the torques, $M$ the inertial matrix, $H$ is the gravity, centrifugal and Coriolis effects vector, $J$ is the Jacobian matrix and $\mathbf{F}_{\mathbf{c}}=\left[\mathbf{F}_{\mathbf{1}}, \mathbf{F}_{\mathbf{2}}, \cdots\right]$ is the vector of the active contact forces that is computed as:

$$
\mathbf{F}_{\mathbf{i}}=\mathbb{W}_{i}^{-1}\left[\begin{array}{ll}
\hat{P}_{i} A_{i} & A_{i}
\end{array}\right] \Omega^{-1} \mathbf{D}_{\mathbf{2}}
$$

with (i) $\hat{P}_{i}$, the screw operator of the contact position $\left(P_{i}\right.$ expressed in the world frame), and (ii) $A_{i}$ the orientation of the contact frame and $\mathbb{W}_{i}=\operatorname{diag}\left(1000 \times \beta_{i}, 1000 \times \beta_{i}, \beta_{i}\right)$ and the $6 \times 6$ matrix:

$$
\Omega=\sum_{i=1}^{N_{c}}\left(\left[\begin{array}{c}
\hat{P}_{i} A_{i} \\
A_{i}
\end{array}\right] \mathbb{W}_{i}^{-1}\left[\begin{array}{ll}
\hat{P}_{i} A_{i} & A_{i}
\end{array}\right]\right)
$$

Note: we assume that the $z$-component is the normal direction of the contact forces.

\section{Cost Function and Constraints}

It is not easy to identify the cost function that will produce human-like walking. In our case, we consider a weighted sum of the motion duration, square torques and jerks as follows:

$$
C=\sum_{i=1}^{N_{p}} a T_{i}+\sum_{i=1}^{N_{d o f}} \int_{0}^{T}\left(b \Gamma_{i}^{2}+c \dddot{q}_{i}^{2}\right) d t
$$

with the weights $a=5, b=1 e^{-2}, c=1 e^{-6}$ that are empirically determined after several simulations. We enforce the feasibility of the motion and the robot's safety by taking into account explicit limits on joint position, velocity and torques with unilateral and friction constraints of the contact forces. We also consider additional constraints in order to make the motion feasible using the balance controller of the HRP-2. 


\section{WALKING MOTION}

We produce three different humanoid walking motions: one is a normal walking (to serve as a comparison reference); the two others have additional constraints on leg motions that are inspired from human leg disabilities.

\section{A. Properties}

The main part of the walking motions is the cyclic part connecting an initial and a final standing posture as presented in Fig. 2. Each motion has 9 phases decomposed into 45 intervals. During the experiments, we perform the cyclic part three times. We also consider the cost function of this cyclic part to be more important than the transition parts, during the optimization process. The step size of the walking motion is $25 \mathrm{~cm}$.

\section{B. Locked Knee}

We study the walking motion that emulates a human's (left) knee sprained and wearing a splint. We can emulate this walking by artificially locking the (left) knee joint of the humanoid robot's leg, i.e. setting it to a constant joint value (ideally in a totally stretched leg configuration). What we observe from human beings in this case is that walking remains possible thanks to the contribution of additional degrees of freedom (namely, in the hip) to move the impaired leg. With the HRP-2, however, the use of the embedded stabilizer for maintaining balance, on the one hand, and the lack of additional degrees of freedom in the legs, on the other hand, do not allow the robot to walk with a fully stretched leg. Therefore, we constrain the left knee joint to be 'locked' at 30 degrees (other values are certainly possible).

\section{Sore Leg}

In another scenario, we emulated the walking motion that is inspired from a human suffering from by a broken or painful (left) foot. To avoid pain, human beings try not to lean too much on the injured foot. In order to emulate such a motion on the humanoid robot, we consider an additional constraint on the contact force of the (left) foot. While the ground reaction force is $586 \mathrm{~N}$ during standby standing posture and even larger during walking, we restrain the contact force of the left foot to be less than $500 \mathrm{~N}$ during the walking motion, which results in an inequality constraint on the left foot's contact force.

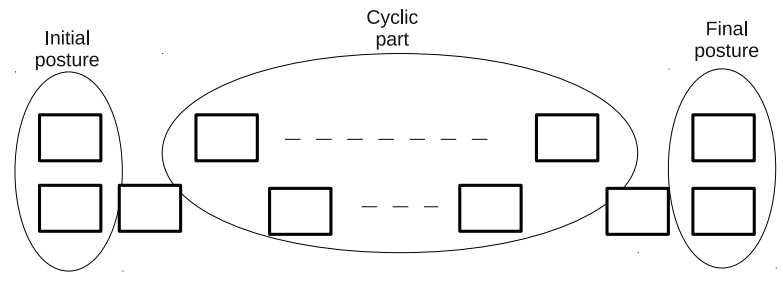

Fig. 2. Representation of the foot stance for the decomposition of the motion walking into cyclic and transition part.

TABLE I

OPTIMIZATION RESULTS.

\begin{tabular}{|c||c|c|c|}
\hline walking motion & $I$ & CPU & $C$ \\
\hline \hline normal & 821 & $3 \mathrm{~h} 27 \mathrm{mn}$ & 166.5 \\
\hline locked knee & 510 & $2 \mathrm{~h} 31 \mathrm{mn}$ & 223.2 \\
\hline sore leg & 1427 & $6 \mathrm{~h} 01 \mathrm{mn}$ & 387.0 \\
\hline
\end{tabular}

\section{Optimization process}

Table I presents the results of the optimization processes to generate the three walking motions. Here, $I, \mathrm{CPU}$ and $C$ are respectively the number of iterations, the computation time, and the final value of the cost function. It appears that the normal walking has the lowest value of the cost function. Table I also shows that the walking motion with a sore leg has a bigger cost function value relatively to the walking motion with a locked knee. Since the cost function reflects, in part, the energy consumption, it is evident that the sore leg walking motion consumes more energy for a humanoid robot.

\section{EXPERIMENTS}

In this section, we present the experimental results that we obtain with the HRP-2 robot. To cope with the flexibilities of the shock absorbing mechanisms located at the ankles, we use the HRP-2 embedded stabilizer. The video of those experiments can be found in [16]. Although the motions are a little awkward, we can recognize the emulated impairment from those walking motions in the video. In order to assess

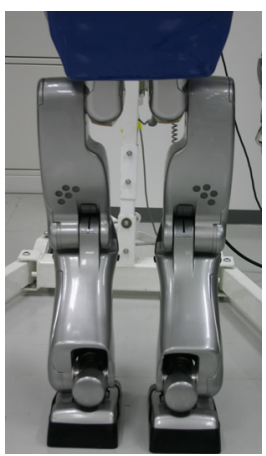

(a) rear view

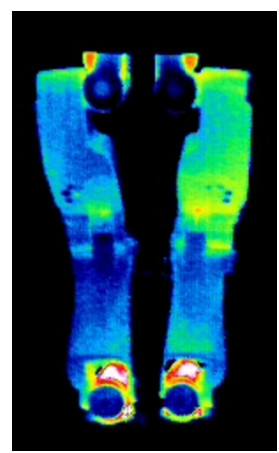

(d) locked knee

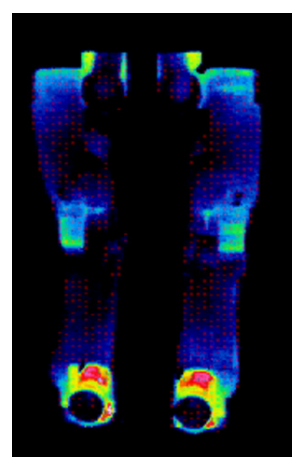

(b) normal walking

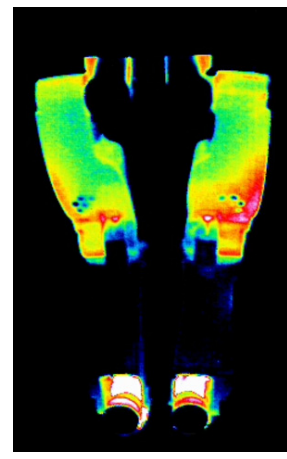

(e) sore leg
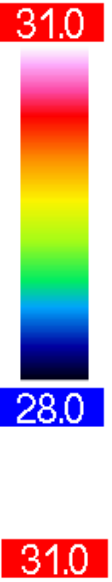

Fig. 3. Infrared view after five rounds of the walking motions (temperatures are expressed in degree Celsius. 

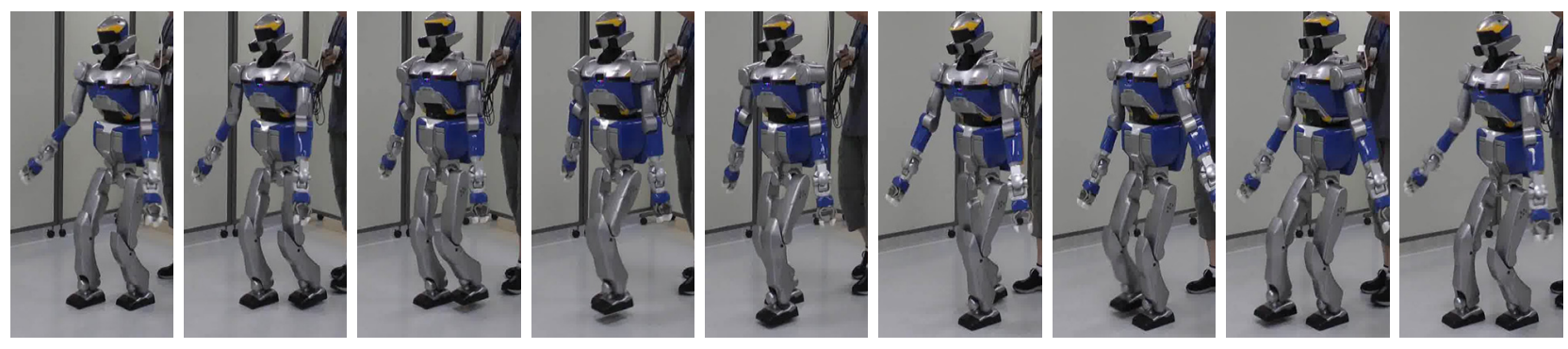

Fig. 4. Normal walk.
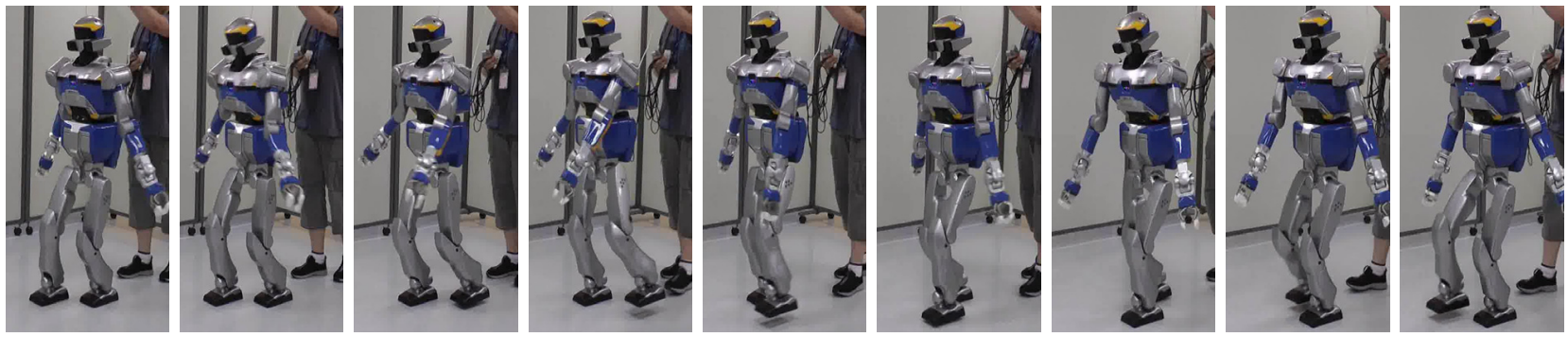

Fig. 5. Walking motion with locked knee.
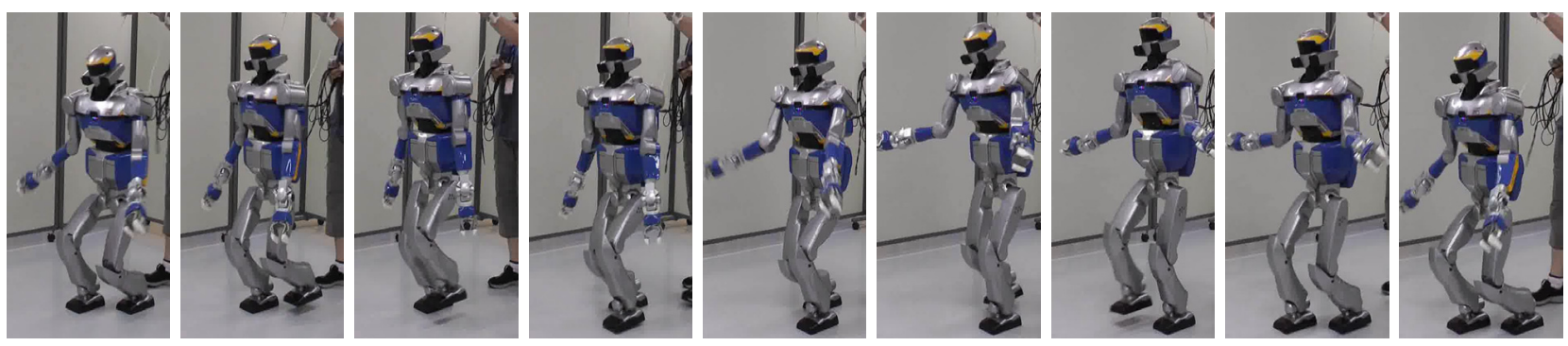

Fig. 6. Walking motion with sore leg.

the energy consumption contribution in the cost function, we use an infrared camera (IR-TCM640 from Jenoptik) and take pictures of the legs of the robot, which reflects the more significant effects (heat) from performing five rounds of the same motion. To get nearly identical thermal conditions, we waited for 45 minutes without using the robot between two

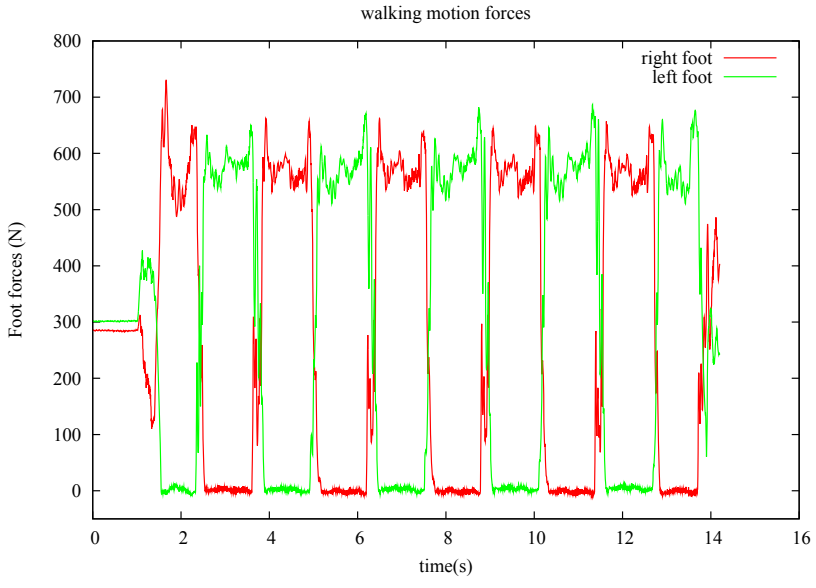

Fig. 7. Foot forces during the normal walking different walking motions (see Fig. 3).

\section{A. Normal Walking}

Snapshots from the normal walking motion are presented in Fig. 4. On Fig. 3(b), we can see from the heat loss temperature that the normal walking does not make excessive

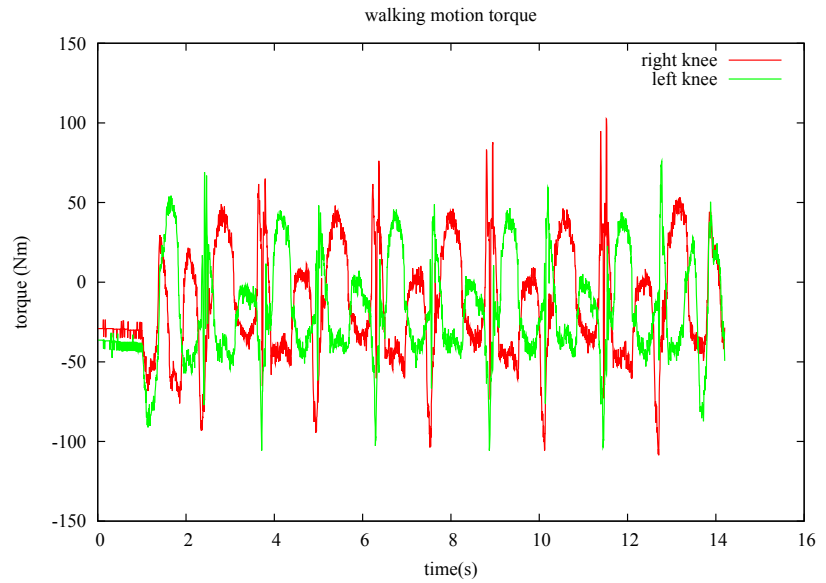

Fig. 8. Knee torques during the normal walking 
energy consumption on any joint. Figures 7 and 8 depict the force applied to the legs and torque estimated from measured motor current. These figures illustrate well the symmetry of the normal walking motion, in terms of contact forces and knee joint torques. Although the motion itself is quite smooth, the walking motion presented in [16] is not really 'human-like'; we discuss more thoroughly this issue in Section V.

\section{B. Locked Knee Walking}

Snapshots from the walking motion with locked left knee are illustrated in Fig. 5. The infrared picture (Fig. 3(d)) shows that the right leg (especially the right knee) compensates the disability of the left knee with additional energy consumption. Nevertheless, whereas the contact forces on the Fig. 9 remain symmetrical, the right knee joint torques is more important relatively to the left one, as seen in Fig. 10; this explains the larger energy consumption in the right leg.

During the locked-knee motion, we did not notice any extra energy consumption in the hip of the HRP-2 robot, whereas for human being this kind of motion leads to fatigue of the hip's muscles. This could be due to a lack of training/solicitation of the corresponding muscles.

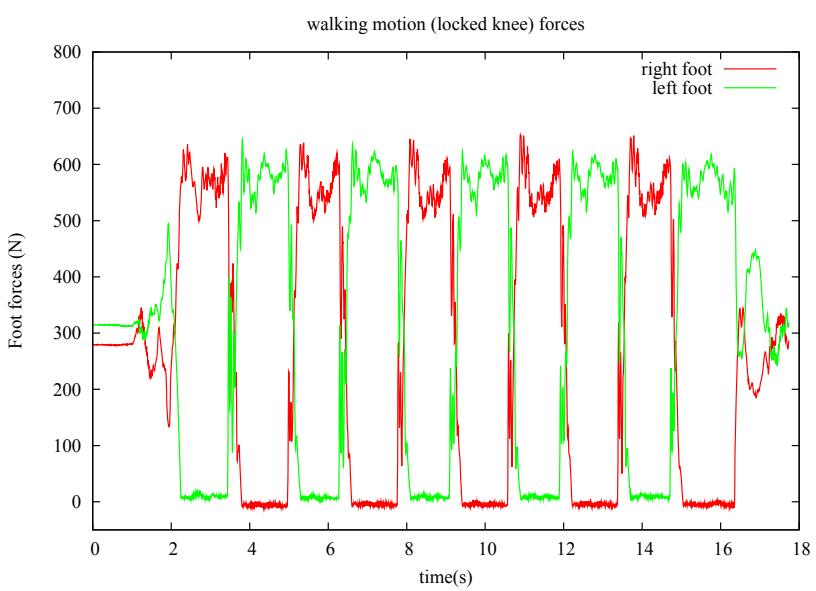

Fig. 9. Foot forces during the locked knee walking

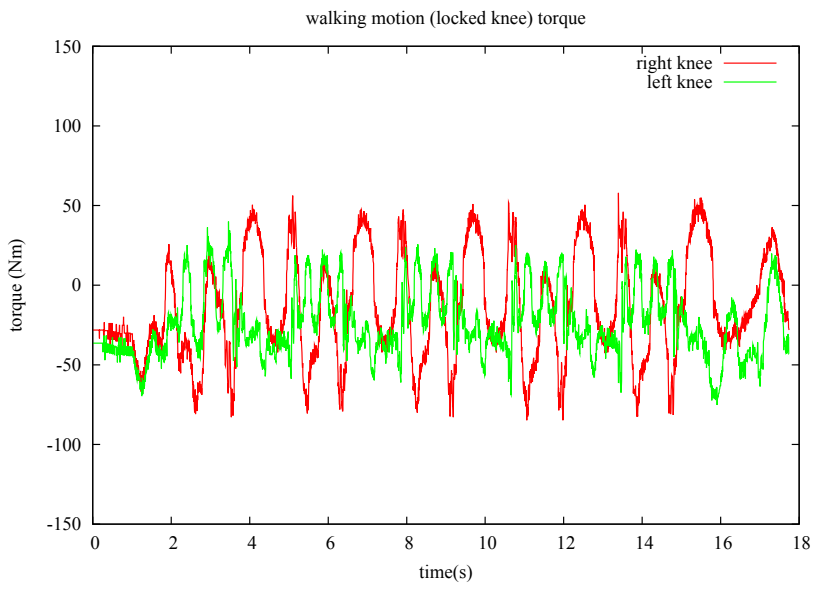

Fig. 10. Knee torques during the locked knee walking

\section{Sore Leg Walking}

Snapshots from the sore leg walking motion are shown in Fig. 6. In Table I, we see that the cost is the highest as compared to the two previous experiments. This is also clearly visible on the infrared monitoring as shown in Fig. 3(e). We can also observe that the contact forces (Fig. 11) are not symmetrical and that the force on the left foot is limited around a mean of $500 \mathrm{~N}$, except the peaks that are caused by non-modeled effects (sharp velocity changes -i.e. foot landing impacts, mechanical flexibilities, etc.) and an open loop playback of the motion. Note the appearance of a preparation phase before the left foot stance phase (during the double support phase) used to accumulate kinetic energy. This preparation was not needed for the previously experimented walking scenarios. Moreover, both the joint torques of the right and left knee are larger than the ones from the previous scenarios, as seen in Fig. 11. This explains that both legs needed extra energy in order to cope with emulating leg impairment of this nature.

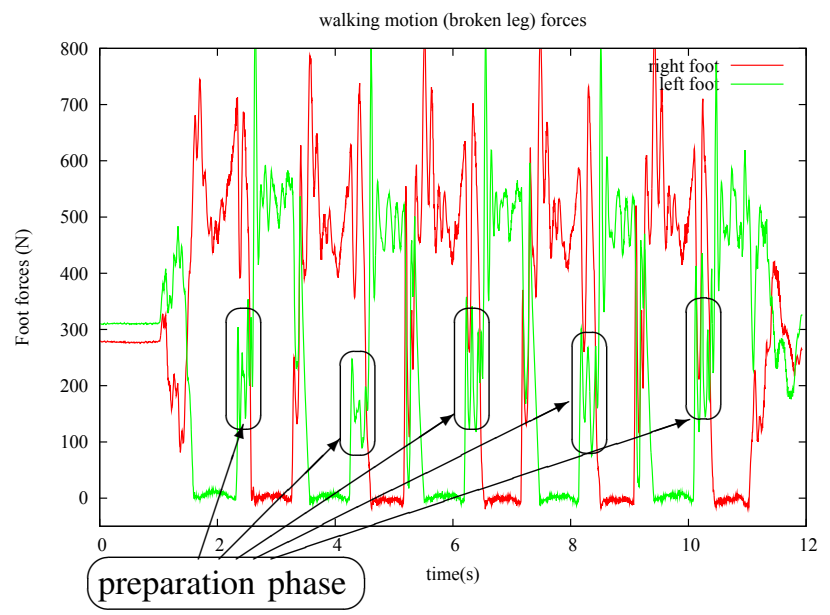

Fig. 11. Foot forces during the sore leg walking. The double support phase is used to accumulate kinetic energy to cope the sore leg.

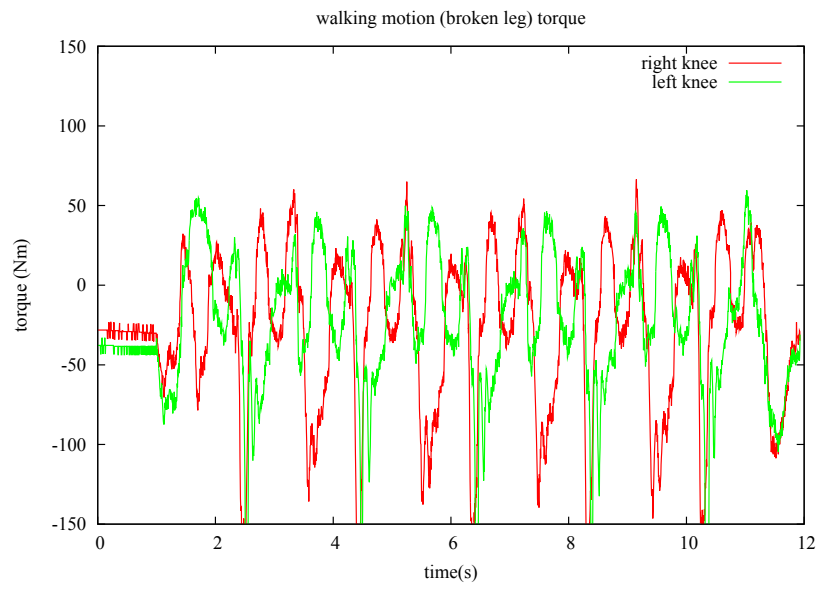

Fig. 12. Knee joint torques during the sore leg walking. 


\section{Discussion}

In this paper, we highlight the benefit of our optimization techniques to emulate very complex motions -such as those resulting from impaired human walks-, provided they can be translated into appropriate robotic constraints. We are certainly aware that the results we obtain for humanoid robots cannot fit exactly those of a human motion under 'somehow' similar constraints.

For instance, we did not observe an augmentation of the hip joint torque with the locked knee while human feel the fatigue at the hip for a similar impairment case, which means the hip is apparently solicited substantially. This is certainly due to the difference, between a biological human and a robotic one, in the actuation physical principles and the body structure, as well as the optimization criteria, etc. Reaching a very close walking behavior is then the aggregate of practical tuning although understanding driving principles may be of some use.

Nevertheless, we believe this work to be a promising step toward motion reproduction or emulation that are close to those of human beings. Future work can exploit explicitly accurate musculo-skeletal models [17], muscle models [18] and muscle fatigue [19] in order to better tune the equivalences. For humanoid robots, we can program many different cost functions, depending on the robot capabilities, performances and the desired task. Nevertheless, it is not easy to guess the cost function that human beings minimize during their motions. In order to get human-like motion, one possible solution could be to integrate human gait patterns extracted from motion capture system. It is also interesting to analyze the human motions in order to reproduce normal walking and also emotional walking of people [20].

\section{CONCLUSION}

In this paper, we illustrated the possibility for emulating walking motions in case of leg impairments by a humanoid robot using our multi-contact motion generation method. Emulating impairments is made simply through additional constraints in the optimization problem. Then, generated motions are successfully experimented with the HRP-2 humanoid robot, which emulated efficiently the impaired behavior. We also discussed the differences between the motions from an energy consumption viewpoint. In future work, we plan to investigate issues related to more complex tasks using implements and deeper understanding of the tuning policies that brings the emulation as close as possible to that of a human motion under various impairment cases.

\section{ACKNOWLEDGMENTS}

This research is partially supported by Japan Society for the Promotion of Science (JSPS) Grant-in-Aid for JSPS Fellows (P09809) and for Scientific Research (B), 22300071, 2010.

\section{REFERENCES}

[1] S. Lengagne, P. Mathieu, A. Kheddar, and E. Yoshida, "Generation of dynamic motions under continuous constraints: Efficient computation using B-Splines and taylor polynomials," in IEEE/RSJ International Conference on Intelligent Robots and Systems (IROS), 2010, pp. 698703.

[2] _- "Generation of dynamic multi-contact motions: 2D case studies," in IEEE-RAS Internationnal conference on Humanoid robots, 2010, pp. 14-20.

[3] S. Kajita, F. Kanehiro, K. Kaneko, K. Fujiwara, K. Yokoi, and H. Hirukawa, "A realtime pattern generator for biped walking," in IEEE International Conference on Robotics and Automation (ICRA)., may 2002.

[4] S. Kajita, F. Kanehiro, K. Kaneko, K. Fujiwara, K. Harada, K. Yokoi, and H. Hirukawa, "Biped walking pattern generation by using preview control of zero-moment point," in IEEE International Conference on Robotics and Automation, vol. 2, september 2003, pp. 1620 - 1626.

[5] A. Herdt, H. Diedam, P.-B. Wieber, D. Dimitrov, K. Mombaur, and M. Diehl, "Online Walking Motion Generation with Automatic Foot Step Placement," Advanced Robotics, vol. 24, pp. 719-737, 2010. [Online]. Available: http://hal.inria.fr/inria-00391408/en/

[6] S. Lengagne, A. Kheddar, and E. Yoshida, "Generation of optimal dynamic multi-contact motions: Application to humanoid robots." IEEE Transactions on robotics, vol. ??, p. ??, 2011, submitted.

[7] R. F. Stengel, Optimal Control and Estimation. Dover, 1994.

[8] O. Von Stryk and R. Bulirsch, "Direct and indirect methods for trajectory optimization," Ann. Oper. Res., vol. 37, no. 1-4, pp. 357373, 1992.

[9] A. Piazzi and A. Visioli, "Global minimum-time trajectory planning of mechanical manipulators using interval analysis," International Journal of Control, vol. 71, pp. 631-652(22), November 1998.

[10] M. D. K. Breteler, S. C. Gielen, and R. G. Meulenbroek, "Endpoint constraints in aiming movements : effects of approach angle and speed," Biological Cybernetics, vol. 85, no. 1, pp. 65 - 75, july 2001.

[11] S. Miossec, K. Yokoi, and A. Kheddar, "Development of a software for motion optimization of robots: Application to the kick motion of the HRP-2 robot," in IEEE International Conference on Robotics and Biomimetics, 2006, pp. 299-304.

[12] A. Piazzi and A. Visioli, "Global minimum-jerk trajectory planning of robot manipulators," in IEEE Transactions on Industrial Electronics, vol. 47, febru 2000, pp. 140-149.

[13] Y. Uno, M. Kawato, and R. Suzuki, "Formation and control of optimal trajectory in human multijoint arm movement," Biological Cybernetics, vol. 6, no. 2, pp. 89-101, juin 1989.

[14] S.-H. Lee, J. Kim, F. Park, M. Kim, and J. E. Bobrow, "Newtontype algorithms for dynamics-based robot movement optimization," in IEEE Transactions on robotics, vol. 21, 2005, pp. 657- 667.

[15] A. Wächter and L. T. Biegler, "On the implementation of a primaldual interior point filter line search algorithm for large-scale nonlinear programming," Mathematical Programming, vol. 106, pp. 22-57, 2006.

[16] [Online]. Available: http://www.youtube.com/watch?v= gx4q23AbWIw

[17] Y. Nakamura, K. Yamane, Y. Fujita, and I. Suzuki, "Somatosensory computation for man-machine interface from motion-capture data and musculoskeletal human model," IEEE Transactions on Robotics, vol. 21, pp. 58-66, 2005.

[18] H. E. Makssoud, D. Guiraud, and P. Poignet, "Enhancement of physiological and mechanical modelling of the skeletal muscle controlled by functional electrical stimulation," in 9th Annual Conference of the International FES Society Bournemouth, UK, september 2004.

[19] M. Papaiordanidou, D. Guiraud, and A. Varray, "Does Central Fatigue Exist Under Low-Frequency Stimulation of a Low Fatigue-Resistant Muscle ?" European Journal Of Applied Physiology, vol. Online preview, pp. 1-9, July 2010.

[20] N. F. Troje, "Decomposing biological motion: A framework for analysis and synthesis of human gait patterns," Journal of Vision, vol. 2, pp. 371-387, 2002. 